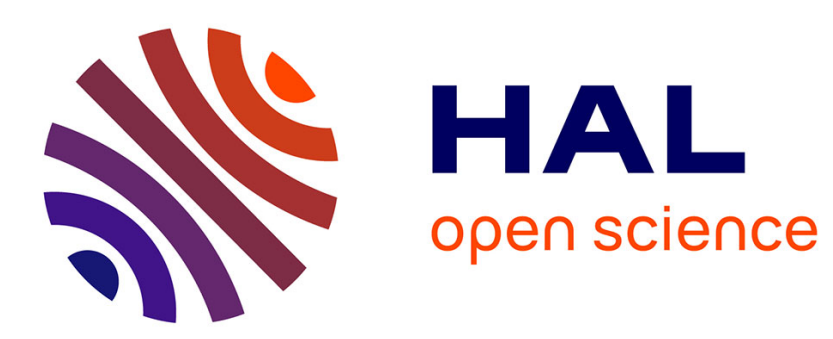

\title{
Phytocides et nanifiants pour l'entretien de coupures de combustible et de pare-feu en région méditerranéenne
}

\author{
Pierre Delabraze
}

\section{To cite this version:}

Pierre Delabraze. Phytocides et nanifiants pour l'entretien de coupures de combustible et de pare-feu en région méditerranéenne. Revue forestière française, 1990, 42 (S), pp.135-139. 10.4267/2042/26127 . hal-03425266

\section{HAL Id: hal-03425266 https://hal.science/hal-03425266}

Submitted on 10 Nov 2021

HAL is a multi-disciplinary open access archive for the deposit and dissemination of scientific research documents, whether they are published or not. The documents may come from teaching and research institutions in France or abroad, or from public or private research centers.
L'archive ouverte pluridisciplinaire HAL, est destinée au dépôt et à la diffusion de documents scientifiques de niveau recherche, publiés ou non, émanant des établissements d'enseignement et de recherche français ou étrangers, des laboratoires publics ou privés. 


\section{PHYTOCIDES ET NANIFIANTS POUR L'ENTRETIEN DE COUPURES DE COMBUSTIBLE ET DE PARE-FEU EN RÉGION MÉDITERRANÉENNE}

Une large gamme de phytocides - herbicides, arboricides, débroussaillants et inhibiteurs de croissance à effets nanifiants - permet de proposer des solutions originales appropriées aux lourdes sujétions que constitue l'entretien des coupures de combustible et des pare-feu en région méditerranéenne.

La simplicité de leur mise en œuvre aurait pu entraîner leur généralisation si ne se posaient certaines appréhensions de nature écologique ou pratique: la formation d'applicateurs conscients des conséquences d'une négligence et, dans certaines circonstances, l'acceptation d'une utilisation mixte de véhicules de surveillance, petits porteurs d'eau, à équiper en conséquence pour ce type de pulvérisation.

En effet, il ne faut pas ignorer les difficultés d'évaluer l'efficacité et la sélectivité des traitements au cours des travaux et d'anticiper, de ce fait, des effets qui vont se développer progressivement. Cela suppose une planification des interventions tenant compte de ces délais.

Il est donc nécessaire d'admettre une grande souplesse de décision à l'échelon de chantiers justiciables d'interventions diversifièes si l'on veut éviter l'arrêt des activités par temps de pluie ou les jours de grand vent ou froids.

Par contre, des matériels spécialisés légers, des lances adaptables à certains petits porteurs d'eau, l'utilisation d'aéronefs agricoles et surtout d'hélicoptères permettent d'envisager l'application de substances aux modes d'action et aux effets aussi particuliers que :

- les destructions sélectives, parfois très spécifiques, de groupes d'espèces dangereuses ou indésirables sur ces coupures de combustible: graminées, herbacées dicotylédones, plantes semi-ligneuses ou ligneuses... laissant le terrain libre à des plantes assurant une couverture du sol moins inflammable;

- l'élimination temporaire de la quasi-généralité de la végétation, à l'exception des arbres, par exemple, tout en conservant le sol en place, structuré et en partie "armé " par les systèmes racinaires subsistants ou dépérissants;

- un arrêt du développement d'une végétation partiellement rabattue par dessèchement ou par dormance prolongée de la dernière élongation terminale.

II faut de plus souligner l'intérêt présenté par l'association de ces techniques chimiques à d'autres, la dévitalisation de certaines espèces végétales ligneuses favorisant l'extension d'herbacées appétentes, ou le dessèchement des charpentes, la conduite du brûlage dirigé. 


\section{STRATÉGIE D'EMPLOI}

Deux voies d'absorption de ces substances chimiques conduisent à distinguer deux stratégies et trois groupes de solutions.

\section{Les herbicides à absorption racinaire quasi exclusive}

Les substances actives sont incorporées au sol, généralement par pulvérisation aqueuse en surface. Le choix de molécules peu solubles sur un sol au pouvoir adsorbant suffisant garantit un positionnement adéquat :

- De la simazine entre 3 et $6000 \mathrm{~g} / \mathrm{ha}$ m.a. (matière active) s'opposant au développement des plantules de petites graines en cours de germination. Soit seule, soit associée à d'autres herbicides plus ou moins mixtes comme l'atrazine, le diuron, le propyzamide... elle constitue une référence intéressante au maintien en l'état d'un pare-feu à sol nu pendant plusieurs mois. La pulvérisation est effectuée sur sol propre.

- Certains granulés à base de chlortiamide ou de dichlobénil n'ont pas donné satisfaction : volatilisation partielle sous les effets de la chaleur, humidité du sol indispensable pour assurer la diffusion et légère toxicité vis-à-vis de résineux en station semi-aride.

- D'autres matières actives sont réservées aux désherbages des zones non cultivées: tébuthiuron, terbuthylazine, thiazafluron ne se trouvent qu'en association avec d'autres molécules. Leur emploi est délicat car ils sont à exclure de toutes les surfaces prospectées par des racines d'arbres implantées dans les zones voisines. Par contre, l'éthydimuron présente une sélectivité intéressante pour les pins, le Pin d'Alep en particulier.

II faut proscrire l'emploi de ces dernières matières actives sur les sols squelettiques fissurés qui les laissent trop facilement migrer, ainsi que sur les reliefs sensibles à une érosion se concentrant sur des zones de colluvionnements.

Les bandes "anti-mégats" en bordure des pistes et autres voies de communications sont traitées en avril-mai : les mélanges d'herbicides sont indispensables pour assurer l'élimination de toute végétation pendant au moins cinq mois (photo 2, p. 139).

\section{Les phytocides à absorption foliaire dominante ou exclusive}

- Le dalapon au printemps, entre 5 et $8000 \mathrm{~g} / \mathrm{ha}$ m.a., est le graminicide strict absorbé par les feuilles et aussi par les racines. Sa sélectivité vis-à-vis des ligneux dépend du pouvoir absorbant des sols, mais, à $5000 \mathrm{~g}$, les Pins manifestent une certaine sensibilité, portée à $10000 \mathrm{~g}$ pour les Chênes en terrain argileux.

- L'hexazinone au printemps à $1800-2000 \mathrm{~g} / \mathrm{ha}$ m.a. est un excellent destructeur d'un grand nombre d'espèces herbacées et de ligneux feuillus. II détruit Ronces, Genêts d'un an, Fougère aigle partiellement, mais son action sur les Bruyères est insuffisante. Sa sélectivité vis-à-vis des pins méditerranéens et d'autres résineux est bonne; Cèdre et Cyprès ne le supportent pas.

- Le glyphosate présente un spectre d'efficacité particulièrement intéressant qu'il est loisible d'exploiter au mieux en modulant des doses recommandées spécifiques entre 1000 et $6000 \mathrm{~g} / \mathrm{ha}$ m.a.. L'adjonction d'un adjuvant (= "surfactant") comme le Genamin T200 BM à 0,5 I par hectolitre de bouillie ramène le coût du débroussaillement dans des limites acceptables pour ces opérations extensives. Monocotylédones, herbacées et dicotylédones sont sensibles à des pulvérisations foliaires ou susceptibles de pénétrer par les lenticelles des écorces minces. Les résineux aoûtés résistent à des doses maximum de $1500 \mathrm{~g} / \mathrm{ha}$.

La pulvérisation dirigée sur les rejets et les petits brins adventifs des souches et collets de brin à écorce épaisse (dès $10 / 15$ ans), de gourmands aussi de ces mêmes portions de tige, entraînent leur destruction, sans conséquence pour les brins: c'est une excellente solution au nettoyage en deux passages et à distance de ces cépées et bouquets d'arbres difficilement accessibles.

- L'association 2,4-D + dichloprop entre 5 et $8000 \mathrm{~g} / \mathrm{ha}$ éq.ac. (= équivalent acide) total en bouillie aqueuse (600 à $1000 \mathrm{l} / \mathrm{ha}$ ou plus selon le volume à traiter) additionnée de 10 à $15 \mathrm{I} / \mathrm{ha}$ d'une huile minérale synergique (diminution de l'évaporation + diminution de la tension superficielle et étalement des gouttelettes + dissociation des cuticules cireuses) élimine les dicotylé- 
Aucun stade de colorations anormales ne se manifeste: cette action persistante, sans agression visuelle, est d'autant plus appréciée que le verdissement des terrains est conservé.

\section{CONCLUSIONS}

C'est volontairement qu'aucune mention de spécialité n'apparaît dans le cours de l'article. De difficiles négociations, en France et bientôt dans le cadre européen, ne prennent guère en considération les besoins des forestiers. Si l'on espère obtenir des homologations acceptables, pour le praticien sylviculteur, les producteurs d'herbicides pour zones non cultivées et les responsables de l'homologation qui se sont attribués les pare-feu ne doivent pas oublier que ces aménagements font partie de la forêt - une "annexion " venant s'ajouter à celle réalisée sur les emprises diverses en forêt comme les lignes électriques - ni ignorer les problèmes pasés par les techniques nouvelles d'aménagement comme celles des coupures de combustible arborées.

Aussi, en l'absence d'une législation cohérente, se trouve-t-on confronté au choix d'herbicides homologués soit pour des "cultures" forestières, soit pour les zones non cultivées!

\section{P. DELABRAZE}

Ancien directeur de la

Station de Sylviculture méditerranéenne

INSTITUT NATIONAL DE LA RECHERCHE AGRONOMIQUE

Avenue A. Vivaldi

84000 AVIGNON

\section{BIBLIOGRAPHIE}

CEMAGREF, INRA. - Phytocides en sylviculture. - CEMAGREF, décembre 1987 (Note technique $n^{\circ} 53$ ). volume $1,120 \mathrm{p}$.

volume 2, 40 tiches "produits".

COLUMA. - Comptes rendus des conférences bisannuelles. - 1979, 1981, 1983, 1985.

DELABRAZE (P.). - Bases biologiques et physiques de la prévention des incendies de forêt. - Paris : CNRS INRA, 1984-1985. - $22 \mathrm{p}$.

VALETTE (J.-C.). - Glyphosate et triclopyr pour l'entretien des pare-teu méditerranéens. In : $18^{\circ}$ Congrès de I'IUFRO, Ljubljana, septembre 1986. $-9 \mathrm{p}$. 
dones herbacées et feuillues. Sa sélectivité n'est acceptable qu'en pulvérisation dirigée ou localisée, sous des tempèratures inférieures à 20-25 au moment du traitement. Son utilisation à proximité de cultures sensibles (certains feuillus comme les peupliers, les vignes...) est à proscrire. Une pulvérisation dirigée vient à bout des rejets et des gourmands (photo 3, p. 139).

- Le triclopyr, débroussaillant des ligneux feuillus, ne présente cependant plus guère de sélectivité en pulvérisation généralisée en plein à $3000 \mathrm{~g} / \mathrm{ha}$ éq. ac.. Il est supporté au tiers de cette dose par les pins et quelques autres résineux aoûtés, et par temps frais. Sur garrigue et maquis, l'addition d'une huile minérale (10-15 I/ha) à l'eau s'avère indispensable: Buis, Genêts, rejets de Chênes et de ligneux feuillus divers, Chêne kermès, Thym, Romarin, Bruyères, Cytise... sont presque détruits dès la première application; ils disparaissent pratiquement après un rappel de traitement effectué dans les deux années qui suivent (photo 1, p. 139).

Le triclopyr est parfois associé au 2,4-D.

D'autres substances pourraient être citées comme l'asulame, matière active spécifique de la Fougère aigle dont la disparition pour 2-4 ans est assurée par pulvérisation de $4000 \mathrm{~g} / \mathrm{ha}$ en été : mais cette plante n'alimente que les feux de fin d'hiver et de printemps, terribles aussi, et la période de traitement est celle d'autres soucis bien souvent.

\section{Nanifiant débroussaillant-inhibiteur de croissance}

Alors que les matières actives précédentes provoquent des défoliations précédées d'un brunissement des feuillages, le fosamine ammonium inhibe l'activité des bourgeons et des méristèmes apicaux provoquant une dormance prolongée, la mort des dernières pousses et l'apparition de feuilles et de rameaux nanifiés sur les verticilles inférieurs des végétations ligneuses dicotylédones traitées. Ainsi, on obtient, par pulvérisation de bouillie aqueuse additionnée d'huile et une dose de 4800 à $7000 \mathrm{~g} / \mathrm{ha}$ éq. ac., une réduction notable de hauteur et de volume des garrigues, des maquis aussi, suivie d'un arrêt pratique d'élongation pendant plus de deux ans et jusqu'à quatre ans. On dispose ainsi d'une formule qui, appliquée précocement sur jeunes rejets courts, favorise la couverture du sol par une phytomasse réduite d'une espèce comme le Chêne kermès, dont la combustibilité vive ne s'exprime que pendant 3-4 mois/an (photo 4, p. 139).

Coupure de combustible arborée en protection d'un lotissement (à draite). Aprẻs broyage de la garrigue à Chêne kermès dominant, la pulvérisation d'un débroussaillant assure le développement d'un tapis de graminées courtes (Brachypode) ; celle d'un nanifiant (partie basse) conserve une couverture minimum du sol par le Chêne kermès (Langlade, Gard).

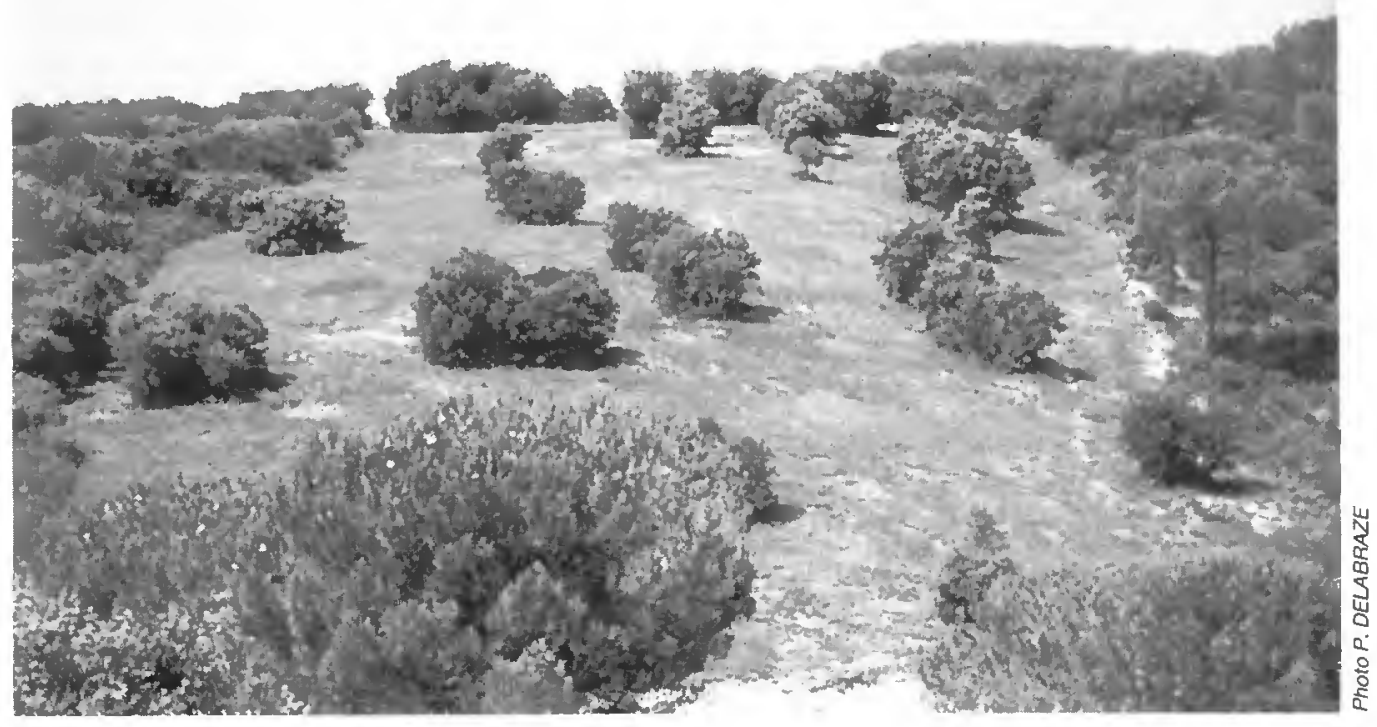




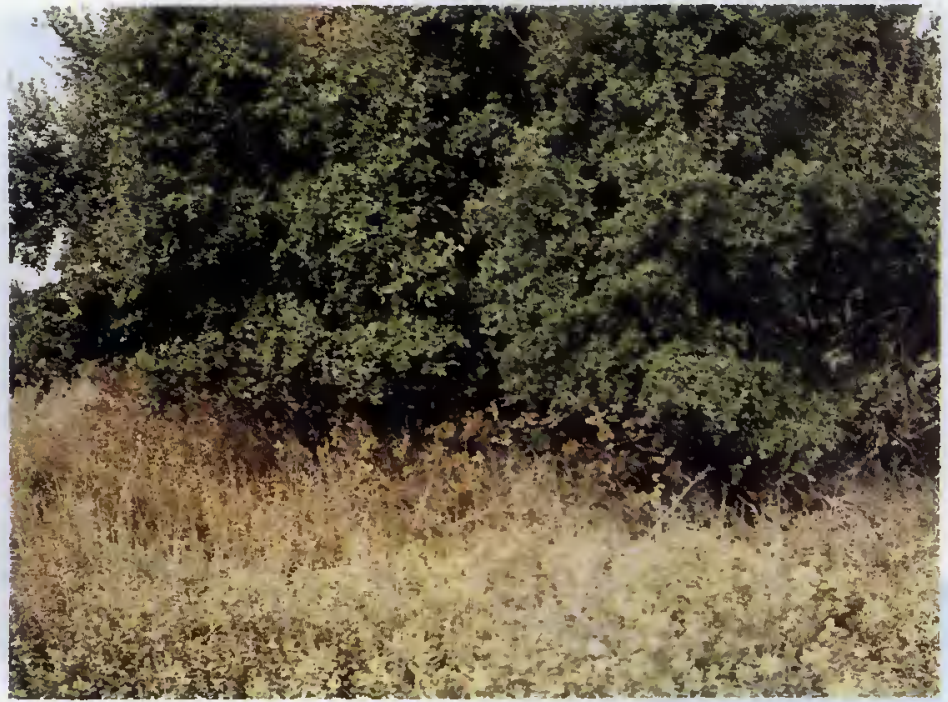

1 - Efficacité du triclopyr (dose forte) sur Chêne kermés et sur petits rejets de Chêne vert, 18 mois après le traitement du 8-10-1987 (Langlade, Gard).

2 - Constitution d'une bande anti-mégots par pulvérisation annuelle de débroussaillants sur les portions * torestiéres * des accotements des routes (Gardanne, Bouches-du-Rhône).

3 - Destruction du Chêne kermès par pulvérisation de phytohormones aux pieds de brins de Chêne vert conservés (Langlade, Gard).

4 - Etfets nanifiants d'une pulvérisation aqueuse à base de fosamine ammonium $(0,8 \%)$ sur Arbousier, 1 an aprés le traitement.
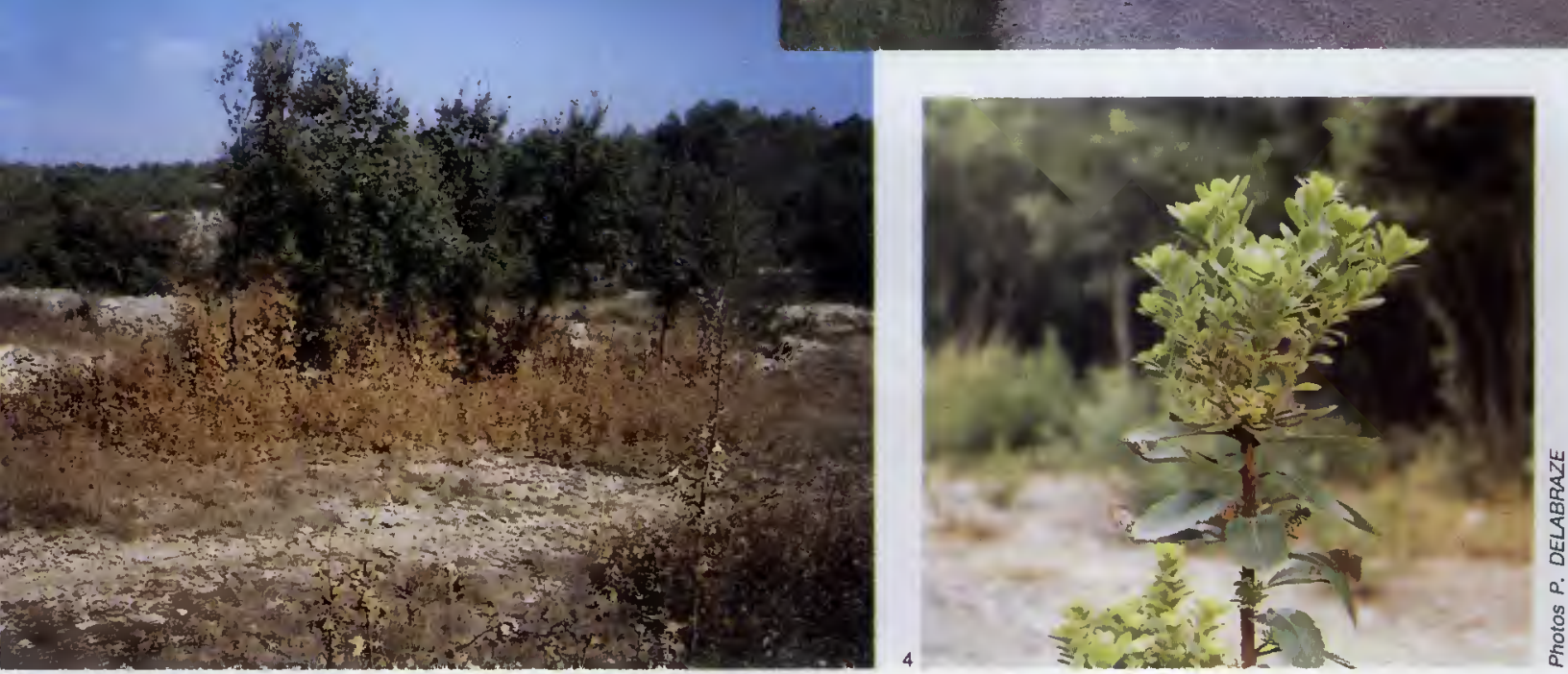